and peroxisome proliferator-activated receptor gamma (PPAR $\gamma$ ) antagonist T0070907 were further determined. To demonstrate the clinical relevance of PPAR $\gamma$, we performed scRNA-seq analysis of tumor biopsies from advanced HCC patients who received anti-PD-1 treatment.

Results We successfully established anti-PD-L1-resistance models, which were accompanied with lower $\mathrm{CD} 8^{+} \mathrm{T}$ cells and $\mathrm{T}$ helper $1\left(\mathrm{~T}_{\mathrm{H}} 1\right)$ cells but higher exhausted $\mathrm{T}$ cells and myeloid-derived suppressor cells (MDSCs). Integrative gene expression analysis showed significant enrichment of PPAR $\gamma$ signaling in PD-L1R tumor cells. Importantly, T0070907 overcame ICB resistance in HCC, which was accompanied with enhanced cytolytic activity and reduced $\mathrm{T}$ cell exhaustion and decreased infiltration of MDSCs. Notably, scRNA-seq profiles of human biopsies uncovered adaptive upregulation of tumor-cell intrinsic PPAR $\gamma$ and re-shaping of T cell exhaustion in non-responders upon anti-PD-1 therapy.

Conclusions Taken together, hepatoma-intrinsic PPAR $\gamma$ activation might be associated with immune evasion and ICB resistance. Pharmacological inhibition of PPAR $\gamma$ sensitized tumors to anti-PD-L1 therapy, thus representing a promising strategy to overcome ICB resistance.

\section{IDDF2020-ABS-0209 ASIAN PREVALENT ALLELE AT ABCB5 SNP RS10254317 ASSOCIATES WITH HEPATOCELLULAR CARCINOMA (HCC) RISK AND ADVERSE CLINICAL OUTCOMES}

${ }^{1}$ Philip Chun Yeung*, ${ }^{1}$ Charing Ching-Ning Chong, ${ }^{2}$ Tan To Cheung, ${ }^{1}$ Kelvin Kwok Chai Ng, ${ }^{1}$ Paul Bo San Lai, 'Siu Tim Cheung. 'The Chinese University of Hong Kong, Hong Kong;

${ }^{2}$ The University of Hong Kong, Hong Kong

\subsection{6/gutjnl-2020-IDDF.39}

Background Ethnic disparities in the prevalence of hepatocellular carcinoma (HCC) continue to exist. Highest age-adjusted HCC incidence rates are recorded in East Asia, and $55 \%$ of HCC cases worldwide are from China. Meanwhile, ABCB5 has been reported to be overexpressed in HCC and associated with poor survival. To evaluate the ethnic differences, allele frequencies of ABCB5 SNP rs10254317 in the local cohort were compared with those from Chinese and Caucasian in 1000 Genomes project, and their clinical implications on HCC patients were studied.

Methods A total of 300 HCC and 300 healthy blood samples $\mathbf{9 9 . 3 \%}$ and $94.7 \%$ Chinese respectively) were prospectively collected with informed consent. All patients had been diagnosed with primary HCC and underwent partial hepatectomy. Clinicopathological information including sex, age, tumour stage and survival outcomes were collected prospectively. Genomic DNA was extracted from blood samples and SNPs were examined. For comparison, genomics data and corresponding clinical information for HCC were obtained from The Cancer Genome Atlas (TCGA). SNP allele frequencies in different populations were obtained from 1000 Genomes Browser by NCBI.

Results Allele frequencies of rs10254317 observed in healthy local cohorts (G: 0.334 vs A: 0.666 ) were comparable to Northern and Southern Chinese (CHB; G: 0.311 vs A: 0.689; CHS; G: 0.286 vs A: 0.714) in 1000 Genomes project, which were significantly different from Caucasian (GBR; G: 0.615 vs A: 0.385$)(\mathrm{p}<0.001)$. Chinese-dominant allele frequency (AA/ AG) associated with higher HCC risk (OR: 2.059, 95\%CI:

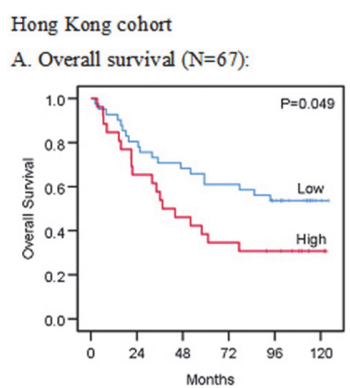

TCGA LIHC cohort

B. Overall survival - Asian $(\mathrm{N}=155)$ :

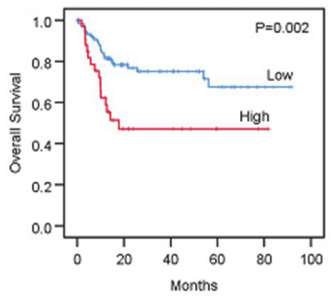

C. Overall survival - Caucasian $(\mathrm{N}=182)$

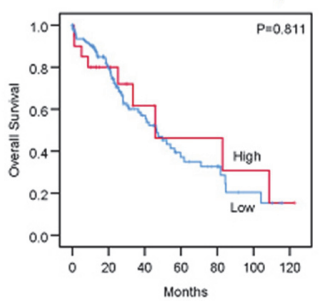

Abstract IDDF2020-ABS-0209 Figure 1 ABCB5 expression associated with poor survival outcomes in Asian HCC patients

1.16-3.67, $\mathrm{p}=0.014)$, advanced tumor stage (OR: 4.514, 95\% CI: $1.02-19.96, p=0.047)$ and presence of venous infiltration (OR: 2.864, 95\%CI: 1.00-8.18, $\mathrm{p}=0.049$ ) from local cohort. TCGA HCC dataset also revealed a disparity in HCC survival outcomes among different populations, as elevated ABCB5 expression levels associated with poor survival in Asian HCCs but not in Caucasian HCCs (figure 1).

Conclusions Dominant allele of rs10254317 in ABCB5 among Chinese associates with risk of HCC and adverse clinical outcomes in HCC patients, which may also contribute to the ethnic disparity in HCC incidence and survival outcomes. Further investigation on SNPs of HCC-related genes with ethnic disparities are warranted.

\section{IDDF2020-ABS-0215 ENHANCER REPROGRAMMING BY SELECTIVE HDAC8 INHIBITION POTENTIATES TUMOR REMISSION AND DURABLE BENEFIT BY PD-L1 BLOCKADE}

${ }^{1}$ Weiqin Yang*, ${ }^{1} Y u$ Feng, ${ }^{1}$ Jingying Zhou, ${ }^{1}$ Otto Ka Wing Cheung, ${ }^{2}$ Feng Wu, ${ }^{3}$ Zhiwu Tan, ${ }^{1}$ Liangliang Xu, ${ }^{4}$ Hanyong Sun, ${ }^{5}$ Yuan Tian, ${ }^{6}$ John Wong, ${ }^{6}$ Paul Bo San Lai, ${ }^{7}$ Stephen Lam Chan, ${ }^{2}$ Wing Hung Chan, ${ }^{8}$ Patrick Tan, ${ }^{3}$ Zhiwei Chen, ${ }^{9}$ Joseph Jao Yiu Sung, ${ }^{10}$ Kevin Yuk Lap Yip, ${ }^{2}$ Ka Fai To, ${ }^{1}$ Alfred Sze Lok Cheng. 'School of Biomedical Sciences, The Chinese University of Hong Kong, Hong Kong; ${ }^{2}$ Department of Anatomical and Cellular Pathology, The Chinese University of Hong Kong, Hong Kong; ${ }^{3}$ AIDS Institute, The University of Hong Kong, Hong Kong; ${ }^{4}$ Department of Liver Surgery, Ren Ji Hospital, School of Medicine, Shanghai Jiao Tong University, China; ${ }^{5}$ Department of Biochemistry and Molecular Biology, Shenzhen University School of Medicine, China; ${ }^{6}$ Department of Surgery, The Chinese University of Hong Kong, Hong Kong; ${ }^{7}$ Department of Clinical Oncology, The Chinese University of Hong Kong, Hong Kong; ${ }^{8}$ SingHealth Duke-NUS Institute of Precision Medicine, National Heart Centre Singapore, Singapore; ${ }^{9}$ Department of Medicine and Therapeutics, The Chinese University of Hong Kong, Hong Kong; ${ }^{10}$ Department of Computer Science and Engineering, The Chinese University of Hong Kong, Hong Kong

\subsection{6/gutjnl-2020-IDDF.40}

Background The insufficient $\mathrm{T}$ cell infiltration into noninflamed tumors such as hepatocellular carcinoma (HCC) restricts the effectiveness of immune-checkpoint blockade (ICB) to a minority of patients. Epigenetic therapy provides new opportunities to rewire cancer transcriptional programs, 
but whether and how selective epigenetic inhibition counteracts the immune-excluded phenotype to sensitize ICB therapy remain incompletely defined. Here, we aimed to investigate the therapeutic efficacy and mechanistic basis of histone deacetylase 8 (HDAC8), a histone H3 lysine 27 (H3K27)specific isoform, in HCC development and ICB responsiveness.

Methods The immune-modulatory and anti-tumor effects of HDAC8 inhibition via a HDAC8-selective inhibitor, PCI34051, were determined in orthotopic HCC mouse models. Molecular mechanisms and functional significance of HDAC8 inhibition were conducted by genome-wide H3K27ac ChIP-seq and RNAseq in HCC patient specimens, cancer cell lines, NODSCID and humanized mouse models. The efficacy of single or combined therapy with anti-programmed death-1-ligand-1 (anti-PD-L1) and PCI34051 was determined in orthotopic and spontaneous HCC mouse models.

Results Pharmacological inhibition of HDAC8 thwarted HCC tumorigenicity in immunocompetent but not immunodeficient mice. The tumor-suppressive effect of PCI34051 was abrogated by $\mathrm{CD}^{+} \mathrm{T}$ cell depletion or regulatory $\mathrm{T}$ cell adoptive transfer. Chromatin profiling of human HDAC8expressing HCCs revealed genome-wide H3K27 deacetylation in 1,251 silenced enhancer-target gene pairs that were enriched in metabolic and immune regulators. Mechanistically, down-regulation of HDAC8 increased global and enhancer levels of $\mathrm{H} 3 \mathrm{~K} 27$ acetylation to reactivate $\mathrm{T}$ celltrafficking chemokine production from HCC cells, thus relieving $\mathrm{T}$ cell exclusion in both NOD-SCID and humanized mouse models. In the HCC preclinical model, selective HDAC8 inhibition significantly increased tumor-infiltrating $\mathrm{CD} 8{ }^{+} \mathrm{T}$ cells and potentiated eradication of established hepatoma by anti-PD-L1 therapy without a sign of toxicity. Importantly, mice treated with HDAC8/PD-L1 co-blockade were protected against subsequent tumor re-challenge with the induction of memory $\mathrm{T}$ cells and remained tumor-free for $\geq 15$ months.

Conclusions Our study demonstrates that selective HDAC8 inhibition elicits effective and durable ICB responses by coopting adaptive immunity via enhancer reprogramming, thereby providing a new strategy for effective combined epigenetic immunotherapy.

\section{Clinical gastroenterology}

\section{IDDF2020-ABS-0014 COMPARISON OF THE EFFICACY OF POVIDONE-IODINE AND NORMAL SALINE WASH IN PREVENTING SURGICAL SITE INFECTIONS IN LAPAROTOMY WOUNDS- RANDOMIZED CONTROLLED TRIAL}

Vinay HG*, Ramesh Reddy. Vydehi Institute of Medical Sciences and Research Centre, India

10.1136/gutjnl-2020-IDDF.41

Background Povidine-Iodine has been utilized as a broad-spectrum antiseptic irrigation in the wound management processes for many years. However, some recent studies showed that the infection rate in laparotomy wounds decreases more by using normal saline.
The study aims to examine the decreasing infection rate in laparotomy wounds by comparing the effectiveness and safety of povidine-iodine solution with normal saline.

Methods The patients undergoing elective laparotomies were included and randomly assigned to 2 groups. In the first group $(90)$, incision wounds were flushed with $5 \%$ povidineiodine solution. In the second group(90), incisions were flushed with $0.9 \%$ normal saline solution. By comparing the infection rates of the wound, outcomes were measured between the two groups.

Results Surgical site infections were seen in 16 of $180(12.5 \%)$ patients in povidine-iodine versus 7 in normal saline groups. The difference in the infection rates in the two studied groups $(\mathrm{p}=0.6)$ has no statistical significance.

Conclusions The infection rate in laparotomy wounds did not increase or decrease when the wound was irrigated with $5 \%$ povidone-iodine solution or with $0.9 \%$ saline solution.

\section{IDDF2020-ABS-0015 PRUCALOPRIDE IN THE TREATMENT OF CHRONIC GERD}

${ }^{1}$ Shivam Shivam*, ${ }^{2}$ Manohar KN. 'Medical Advisor, Boehringer Ingelheim, India; ${ }^{2}$ Consultant Physician, Manipal Hospitals Bangalore, India

\subsection{6/gutjnl-2020-IDDF.42}

Background Treatment of persistence of gastroesophageal reflux disease (GERD) symptoms, despite readily available medications and lifestyle advocation is challenging. Prucalopride, a selective high-affinity serotonin $\left(5-\mathrm{HT}_{4}\right)$ receptor agonist, has been a good therapeutic alternative. Data from large randomized, controlled clinical trials indicate that it is effective in chronic constipation and offers a new therapeutic alternative for those whose condition fails to respond to conventional laxatives. Clinical use of Prucalopride in GERD has been of immense use in patients refractory to the conventional pharmacological treatments.

Methods A 45-year-old type 2 diabetic, chronically constipated male gastroesophageal reflux disease-patients presented to our outpatient department with reflux symptoms and an increased number of reflux episodes. Gastroesophageal reflux was detected by multichannel impedance (MII) monitoring. He had been prescribed Pantoprazole and domperidone for his symptoms in the past but did not get relieved. We treated him with prucalopride $(2 \mathrm{mg} / \mathrm{day})$. The second MII-monitoring after the initiation of prucalopride $2 \mathrm{mg}$ per day (plus pantoprazole $40 \mathrm{mg}$ per day) showed an overall decrease in reflux episodes.

Results Numbers of all reflux episodes, as well as non-acid reflux episodes, were reduced in the patients. The objective findings were concordant with subjective reports of symptomatic relief. There were no major adverse events during therapy with prucalopride.

Conclusions Administration of prucalopride showed promising results in the treatment of persisting or weakly and/or non-acid reflux episodes in this chronically constipated patient. Therefore, prucalopride can be regarded as a good therapeutic option in the treatment of standard proton pump inhibitor-persistent reflux in the chronically constipated patients. 